\title{
ESTUDO CINÉTICO E DE EQUILÍBRIO DE ADSORÇÃO PARA REMOÇÃO DE FENOL EM SOLUÇÕES AQUOSAS UTILIZANDO CARVÃO ATIVADO COM CO
}

\author{
T. E. P. SILVA ${ }^{1 *}$, J. J. SILVA, J. H. L. $\operatorname{SANTOS}^{1}$, C. M. B. M. BARBOSA ${ }^{1}$ e M. M. M. B. \\ DUARTE $^{1}$ \\ ${ }^{1}$ Universidade Federal de Pernambuco, Departamento de Engenharia Química. \\ *E-mail para contato: thiago_pe @ig.com.br
}

\begin{abstract}
RESUMO - O fenol está presente em efluente da indústria do petróleo devendo ser removido antes de seu descarte, sendo o processo adsortivo uma alternativa para essa remoção. $\mathrm{O}$ objetivo do trabalho foi avaliar o uso do carvão da palha de coqueiro ativado com $\mathrm{CO}_{2}$ para remoção de fenol em soluções. A evolução cinética inicial foi rápida até 100 min., atingindo o equilíbrio em 360 min. O modelo pseudo $n$ ordem foi o que melhor se ajustou aos dados experimentais conforme o teste $F$, para um nível de $95 \%$ de confiança. Com base nos modelos de Weber-Morris e Boyd, o processo adsortivo é controlado por mais de uma etapa, incluindo difusão intrapartícula e difusão através do filme. Para o equilíbrio de adsorção o modelo de Fritz-Schlunder apresentou melhor ajuste aos dados experimentais quando comparado, pelo teste $F$, ao modelo LangmuirFreundlich. A capacidade adsortiva máxima foi de 55,8 $\pm 6,9 \mathrm{mg} \cdot \mathrm{g}^{-1}$ demostrando ser um potencial adsorvente para tratamento de efluentes contendo fenol.
\end{abstract}

\section{INTRODUÇÃO}

As refinarias de petróleo geralmente utilizam grandes volumes de água, especialmente para manutenção de equipamentos, sistemas de refrigeração, dessalinização e vaporização. As características das águas residuais variam de acordo com alguns fatores tais como: a natureza do processo, o tipo de matéria-prima e as etapas de transformação utilizadas (SANTO, 2010).

Segundo o mesmo autor, as refinarias podem gerar efluentes com diversos tipos de contaminantes, por exemplo, metais pesados, sólidos em suspensão, fenóis e compostos sulfurados. Esses efluentes necessitam de tratamentos adequados para não comprometerem a qualidade dos corpos hídricos receptores.

O fenol é considerado um poluente perigoso presente em efluentes de refinaria, devido a sua toxicidade, mesmo em baixas concentrações. Sua presença em águas naturais pode ocasionar a formação de outras substâncias tóxicas (BUSCA et al., 2008).

Várias tecnologias para tratamento de efluentes estão disponíveis, tais como: floculação (PARSONS; JEFFERSONS, 2006), filtração (ZOUBOULIS et al., 2002), lodos ativados (PALA; 


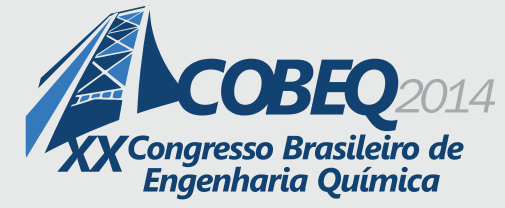

TOKAT, 2002). Cada uma delas apresenta uma série de vantagens e desvantagens. Para a definição do tipo de tratamento a ser utilizado devem ser levados em consideração parâmetros como: eficiência, segurança, simplicidade, formação de lodo, custos de construção e operação, espaço requerido e impacto no meio receptor. Esses métodos convencionais nem sempre são convenientes, completamente eficientes ou ainda apresentam custos elevados (BHATNAGAR; SILLANPAA, 2010; MITTAL et al., 2010).

Dentre os processos de tratamento o processo adsortivo se destaca por ser de simples aplicação, possuir possibilidade de recuperação do adsorvato e adsorvente, além de ser de baixo custo, evita a utilização de solventes tóxicos e o processo não é afetado por substâncias tóxicas (SOTO et al., 2011).

O objetivo do trabalho foi utilizar o carvão da palha de coqueiro ativado com $\mathrm{CO}_{2}$, como adsorvente, para remoção de fenol em soluções, avaliando a cinética e o equilíbrio de adsorção.

\section{METODOLOGIA}

Os experimentos de adsorção foram realizados em banho finito, e ao final de cada ensaio os teores foram quantificados antes e após a execução dos experimentos pela técnica Espectrometria UV-VIS, em 270nm. Foram realizados ensaios em branco, seguindo-se o mesmo procedimento das amostras.

A quantidade adsorvida por massa de adsorvente $\left(q, \mathrm{mg} \cdot \mathrm{g}^{-1}\right)$ foi calculada utilizando a Equação 1.

$q=\frac{\left(c_{0}-c_{f}\right) V}{m}$

Sendo, $C_{o}$ e $C_{f}$ a concentração inicial e final respectivamente $\left(\mathrm{mg} . \mathrm{L}^{-1}\right), V$ o volume da solução (L) e $m$ a massa de adsorvente (g).

O carvão foi ativado em forno elétrico (Marca Lindberg, Blue M), com fluxo de nitrogênio seguido por fluxo de $\mathrm{CO}_{2}$ à vazão de $100 \mathrm{~mL} \mathrm{~min}^{-1}$, por 1 hora cada. $\mathrm{O}$ material foi classificado com uma série de peneiras de Tyler na granulometria $(\mathrm{G})<0,090 \mathrm{~mm}$.

As condições operacionais do sistema para o estudo cinético e de equilíbrio foram definidas em estudo anterior, no qual foram definidas as condições que forneceu maior capacidade adsortiva.

\subsection{Estudo Cinético}

O estudo cinético foi realizado colocando-se em contato $0,1 \mathrm{~g}$ do adsorvente na granulometria $<0,090 \mathrm{~mm}$ com $25 \mathrm{~mL}$ da solução fenólica $\left(100 \mathrm{mg} \cdot \mathrm{L}^{-1}\right)$ no $\mathrm{pH}$ 6. O tempo de contato vaiou de 0 a 600 min e as misturas permaneceram sob agitação constante de $300 \mathrm{rpm}$. Foram utilizados os modelos cinéticos de pseudo-primeira ordem (Equação 2), pseudo-segunda 
ordem (Equação 3) e pseudo $n$ ordem (Equação 4), além dos modelos de difusão intrapartícula de Weber-Morris (Equação 5) e de Boyd (Equações 6 e 7) para avaliação do mecanismo de adsorção (Tabela 1).

Tabela 1 - Modelos cinéticos de adsorção.

\begin{tabular}{|c|c|c|c|}
\hline Modelo cinético & Equações & Constantes e variáveis & \\
\hline $\begin{array}{l}\text { Pseudo-primeira } \\
\text { ordem }\end{array}$ & $\frac{d q_{t}}{d t}=k_{1}\left(q_{e}-q_{t}\right)$ & $\begin{array}{l}k_{l} \text { é a constante da taxa de adsorção de pseudo-primeira } \\
\text { ordem }\left(\text { min }^{-1}\right) \text {. } \\
q_{e} \text { e } q_{t} \text { são a quantidade de adsorvato em mg adsorvido } \\
\text { por grama de adsorvente no equilíbrio e no tempo } t \text {, } \\
\text { respectivamente }\left(\mathrm{mg}^{\left.-\mathrm{g}^{-1}\right)} \text {. }\right.\end{array}$ & (2) \\
\hline $\begin{array}{l}\text { Pseudo-segunda } \\
\text { ordem }\end{array}$ & $\frac{d q_{t}}{d t}=k_{2}\left(q_{e}-q_{t}\right)^{2}$ & $\begin{array}{l}k_{2} \text { é a constante da taxa de adsorção da pseudo-segunda } \\
\text { ordem }\left(\mathrm{g} \cdot \mathrm{mg}^{-1} \cdot \mathrm{min}^{-1}\right) \text {. }\end{array}$ & (3) \\
\hline Pseudo $n$ ordem & $\frac{d q_{t}}{d t}=k_{n}\left(q_{e}-q_{t}\right)^{n}$ & $\begin{array}{l}K_{n} \text { é a constante da velocidade de reação do modelo } \\
\text { pseudo } n \text { ordem. } \\
n \text { é a ordem de reação }\left(\mathrm{kg}^{\mathrm{n}-1} \mathrm{~g}^{1-\mathrm{n}} \min ^{-1}\right) \text {. }\end{array}$ & (4) \\
\hline Weber-Morris & $q_{t}=k_{d i f} t^{1 / 2}+C$ & $\begin{array}{l}k_{i d} \text { a constante de difusão intrapartícula }\left(\mathrm{mg} \mathrm{g}^{-1} \min ^{-1 / 2}\right) \\
C\left(\mathrm{mg} \cdot \mathrm{g}^{-1}\right) \text { é uma constante relacionada com a resistência } \\
\text { à difusão. }\end{array}$ & (5) \\
\hline Boyd & $\begin{array}{l}B t=-0.4977-\ln (1-F) \\
F=\frac{q_{t}}{q_{e}}\end{array}$ & $F$ pode ser calculado pela Equação 7. & $\begin{array}{l}\text { (6) } \\
\text { (7) }\end{array}$ \\
\hline
\end{tabular}

\subsection{Estudo do Equilíbrio}

Para o estudo de equilíbrio foram realizados experimentos no tempo estabelecido no estudo cinético, utilizando $0,1 \mathrm{~g}$ do adsorvente em contato com $25 \mathrm{~mL}$ da solução fenólica nas concentrações de $5 ; 15 ; 35 ; 50 ; 75 ; 100 ; 120 ; 150 ; 200 ; 300$ mg.L $\mathrm{L}^{-1}$, em pH 6 a 300 rpm. Foram aplicados os modelos de adsorção de Langmuir-Freundlich (Equação 8) e Fritz-Schlunder (Equação 9) para avaliar os dados de equilíbrio do processo.

$$
q_{e}=\frac{q_{\max } K_{L F} C_{e}^{1 / n}}{1+K_{L F} C_{e}^{1 / n}}
$$

em que, $q_{\max }$ é a capacidade máxima adsortiva $\left(\mathrm{mg} \mathrm{g}^{-1}\right), K_{L F}$ é a constante de LangmuirFreundlich e $C_{e}$ a concentração do adsorvato no equilíbrio (mg.L $\mathrm{L}^{-1}$ ).

$$
q_{e}=\frac{K_{F S} C_{e}^{b_{1}}}{1+a C_{e}^{b_{2}}}
$$

$K_{F S}$ e $a$ são as constantes de Fritz-Schlunder e $b_{1}$ e $b_{2}$ são os fatores de heterogeneidade. 


\section{RESULTADOS E DISCUSSÃO}

\subsection{Estudo cinético}

Pode ser observada, na Figura 1, a curva de evolução cinética da adsorção do fenol e os ajustes não lineares dos modelos cinéticos de pseudo-primeira ordem e pseudo-segunda ordem e pseudo $n$ ordem.

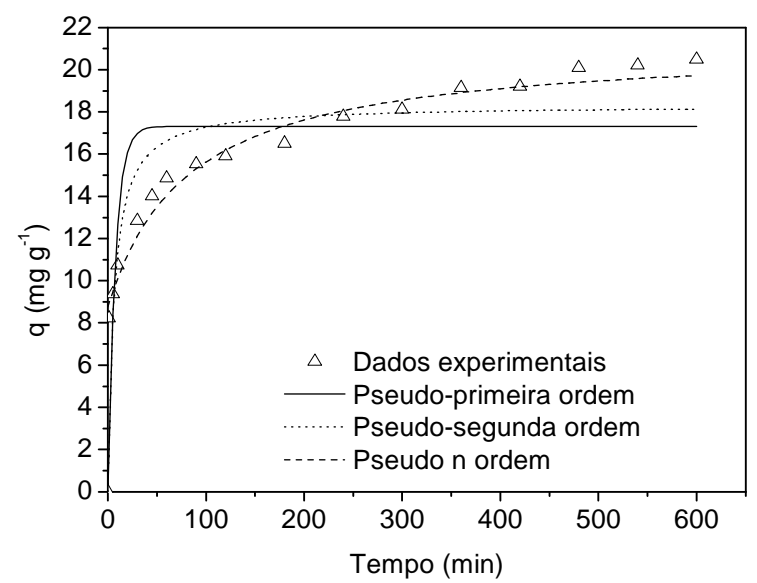

Figura 1 - Evolução cinética da adsorção do fenol em carvão ativado com $\mathrm{CO}_{2}$ e os ajustes não lineares dos modelos cinéticos. $\mathrm{pH}=6,0, \mathrm{C}_{0}$ de $100 \mathrm{mg} . \mathrm{L}^{-1}, \mathrm{C}_{\text {ads }} \mathrm{de} 4 \mathrm{~g} \mathrm{~L}{ }^{-1}, \mathrm{G}<0,090 \mathrm{~mm}$ e $300 \mathrm{rpm}$.

A evolução cinética inicial foi acentuada, com a maior remoção em até 120 minutos. A etapa seguinte foi lenta com equilíbrio em torno de 480 minutos. Os parâmetros dos modelos cinéticos calculados estão disponíveis na Tabela 2.

Tabela 2 - Parâmetros dos modelos cinéticos calculados para o carvão utilizado na adsorção de fenol.

\begin{tabular}{|c|c|c|}
\hline Modelos & Parâmetros & Valores \\
\hline \multirow{4}{*}{${ }^{1}$ Pseudo-primeira ordem } & $q_{\text {ecalc. }}\left(\mathrm{mg} \cdot \mathrm{g}^{-1}\right)$ & $17,31 \pm 0,78$ \\
\hline & $k_{l}\left(\min ^{-1}\right)$ & $0,13 \pm 0,04$ \\
\hline & $S_{R}^{2}\left(\mathrm{mg}^{2} \cdot \mathrm{g}^{-2}\right)$ & 115,33 \\
\hline & $R^{2}$ & 0,735 \\
\hline \multirow{4}{*}{${ }^{2}$ Pseudo-segunda ordem } & $q_{\text {ecalc. }}\left(\mathrm{mg} \cdot \mathrm{g}^{-1}\right)$ & $18,32 \pm 0,72$ \\
\hline & $k_{2}\left(\mathrm{~g} \cdot \mathrm{mg}^{-1} \cdot \mathrm{min}^{-1}\right)$ & $0,009 \pm 0,003$ \\
\hline & $S_{R}^{2}\left(\mathrm{mg}^{2} \cdot \mathrm{g}^{-2}\right)$ & 69,81 \\
\hline & $R^{2}$ & 0,840 \\
\hline \multirow{5}{*}{${ }^{3}$ Pseudo $n$ ordem } & $q_{\text {ecalc }}\left(\mathrm{mg} \cdot \mathrm{g}^{-1}\right)$ & $21,20 \pm 0,47$ \\
\hline & $k n\left(\mathrm{~g}^{\mathrm{n}-1} \cdot \mathrm{mg}^{1-\mathrm{n}} \cdot \mathrm{min}^{-1}\right)$ & $4,42 \cdot 10^{-5} \pm 1,44 \cdot 10^{-5}$ \\
\hline & $N$ & $2,70 \pm 0,05$ \\
\hline & $S_{R}^{2}\left(\mathrm{mg}^{2} \cdot \mathrm{g}^{-2}\right)$ & 4,70 \\
\hline & $R^{2}$ & 0,976 \\
\hline \multirow{4}{*}{ Teste $F$} & $F_{\text {cal }(1 / 2)}$ & 1,65 \\
\hline & $F_{\text {cal }(1 / 3)}$ & 24,53 \\
\hline & $F_{c a l(2 / 3)}$ & 14,85 \\
\hline & $F_{t a b}$ & 2,14 \\
\hline
\end{tabular}


A partir dos resultados do teste $F$, considerando-se um nível de confiança de $95 \%$ (Tabela 1 ), o modelo pseudo $n$ ordem $\left(F_{c a l}>F_{t a b}\right)$ foi o que melhor se ajustou aos dados experimentais.

O Modelo de difusão intrapartícula de Morris $\left(q_{t} v s . t^{1 / 2}\right)$ está apresentado na Figura 2.

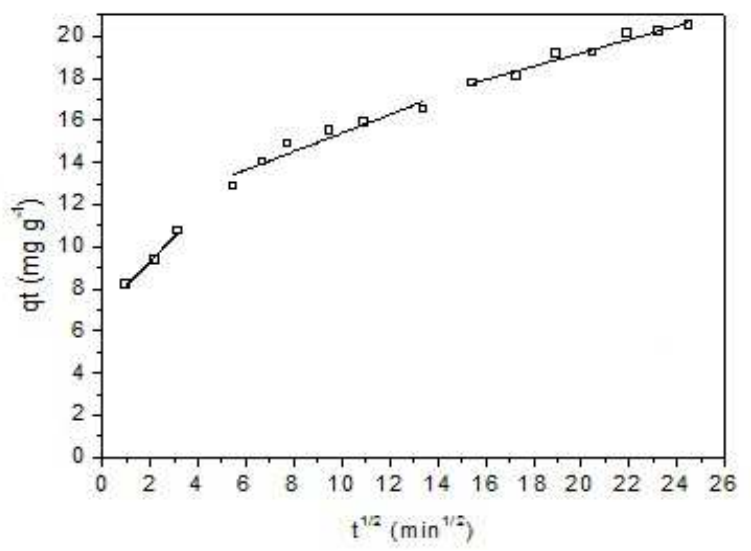

Figura 2 - Modelo de difusão intrapartícula de Weber-Morris.

Devido à multilinearidade dos dados, e o gráfico não passar pela origem, Figura 2, duas ou mais etapas controlam o processo, conforme Abdelwahad e Amin (2014) e Weber et al., 1963 apud Fierro et al., 2008. Logo, deve-se avaliar cada região linear separadamente (Tabela 2).

Tabela 2 - Parâmetros do modelo de difusão intrapartícula de Weber Morris.

\begin{tabular}{|c|c|c|c|}
\hline Parâmetros & $\begin{array}{c}k_{\text {dif }} \\
\text { mg. }\left(\mathrm{g} \cdot \mathrm{min}^{0.5}\right)^{-1}\end{array}$ & $\begin{array}{c}C \\
\left(\mathrm{mg} \mathrm{g}^{-1}\right)\end{array}$ & $R^{2}$ \\
\hline Região 1 & $1,154 \pm 0,150$ & $6,988 \pm 0,348$ & 0,966 \\
\hline Região 2 & $0,438 \pm 0,069$ & $11,025 \pm 0,645$ & 0,887 \\
\hline Região 3 & $0,319 \pm 0,028$ & $12,816 \pm 0,578$ & 0,955 \\
\hline
\end{tabular}

Pode-se observar na Tabela 2, que a constante de difusão intrapartícula vai diminuindo com o decorrer do tempo, isto indica uma diminuição da difusão intrapartícula à medida que o fenol é adsorvido pelo carvão, devido à baixa concentração de fenol na solução, conforme Arthy e Saravanakumar (2013).

$\mathrm{Na}$ Figura 3, pode-se observar o modelo cinético de Boyd ajustado aos dados experimentais. 


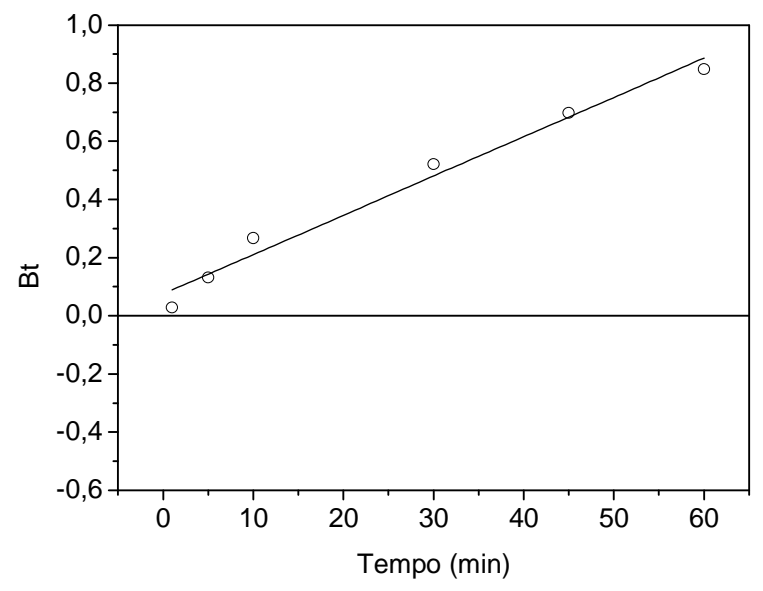

Figura 3 - Modelo de Boyd.

Pode-se observar na Figura 3 que o gráfico não passa pela origem, indicando que dentre as etapas controladoras do processo, está à difusão através do filme, confirmando os resultados obtidos pelo modelo de Weber-Morris.

\subsection{Estudo do equilíbrio}

As isotermas de adsorção e os ajustes não lineares dos modelos de Langmuir-Freundlich e Fritz-Schlunder estão apresentadas na Figura 4.

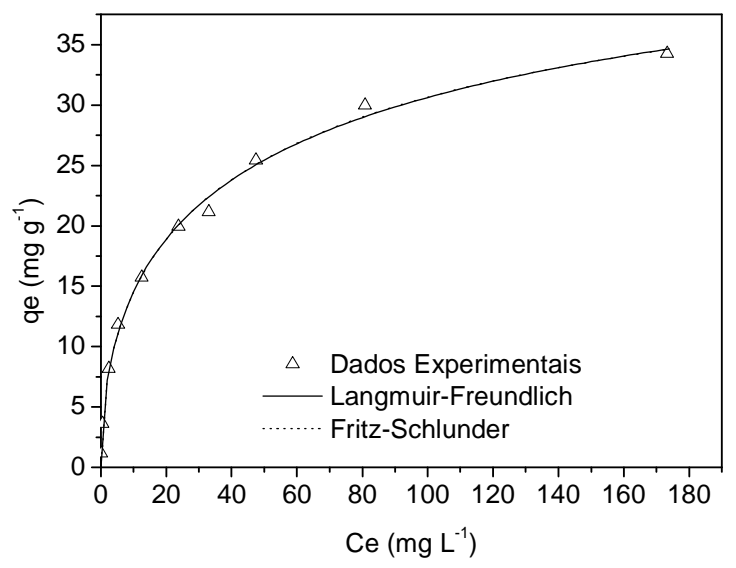

Figure 4 - Isoterma de adsorção em sistema de banho finito, com os ajustes não lineares dos modelos.

Na Tabela 3 pode-se observar os parâmetros dos modelos de equilíbrio de adsorção calculados para o adsorvente. 
Tabela 3 - Parâmetros dos modelos de equilíbrio.

\begin{tabular}{|c|c|c|}
\hline Modelos & Parâmetros & $\mathrm{C}_{\mathrm{CO} 2}$ \\
\hline \multirow{5}{*}{ Langmuir- Freundlich } & $\mathrm{q}_{\text {máx }}\left(\mathrm{mg} \cdot \mathrm{g}^{-1}\right)$ & $55,8 \pm 6,9$ \\
\hline & $B\left(L . g^{-1}\right)$ & $0,10 \pm 0,01$ \\
\hline & $n$ & $1,86 \pm 0,16$ \\
\hline & $S_{R}^{2}\left(\mathrm{mg}^{2} \cdot \mathrm{g}^{-2}\right)$ & 3,677 \\
\hline & $R^{2}$ & 0,996 \\
\hline \multirow{7}{*}{ Fritz-Schlunder } & $K_{F S}\left(\left(m g \cdot g^{-1}\right)\left(m g \cdot d m^{-3}\right)^{-b l}\right)$ & $5,6 \pm 0,9$ \\
\hline & $a\left(\left(m g \cdot d m^{-3}\right)^{-b 2}\right)$ & $0,077 \pm 0,001$ \\
\hline & $b_{1}$ & $0,52 \pm 0,14$ \\
\hline & $b_{2}$ & $1,56 \pm 0,19$ \\
\hline & $S_{R}^{2}\left(\mathrm{mg}^{2} \cdot \mathrm{g}^{-2}\right)$ & 3,673 \\
\hline & $R^{2}$ & 0,995 \\
\hline & $F_{c a l}$ & 1,00 \\
\hline
\end{tabular}

A partir dos resultados encontrados por meio do teste $F$, $\left(\mathrm{F}_{\text {cal }}(\right.$ Tabela 7$)<\mathrm{F}_{\text {tab }}(3,18)$ ), para um nível de $95 \%$ de confiança não existe diferença significativa entre os dois modelos avaliados.

Observa-se na Tabela 3, que na isoterma de Langmuir-Freundlich, o valor de $n$ é maior que 1, isto indica uma adsorção favorável do soluto no carvão conforme Singh et al. (2008).

A capacidade adsortiva máxima $\left(\mathrm{q}_{\max }\right)$ do carvão utilizado foi de 55,8 $\pm 6,9 \mathrm{mg} \cdot \mathrm{g}^{-1}$. Este valor é superior ao encontrado por Singh et al., (2008) que utilizaram o carvão ativado da casca do coco para remoção de fenol, obtendo $\mathrm{q}_{\max }$ de $34 \mathrm{mg}^{\mathrm{g}} \mathrm{g}^{-1}$; e inferior ao encontrado por Rodrigues et al., (2011) que utilizaram o carvão ativado do caroço de abacate, com $\mathrm{q}_{\max }$ de 90 $\mathrm{mg} \cdot \mathrm{g}^{-1}$, no entanto utilizaram concentração inicial de $600 \mathrm{mg} \cdot \mathrm{L}^{-1}$.

\section{CONCLUSÃO}

Diante do exposto o carvão da palha do coqueiro ativado com $\mathrm{CO}_{2}$ apresentou-se como uma alternativa técnica viável para a remoção de fenol em soluções aquosas.

\section{AGRADECIMENTOS}

A Elephant Indústria Química Ltda. por fornecer o carvão utilizado no trabalho e ao PRH28/ANP e Petrobrás pelo apoio e bolsa concedida.

\section{REFERÊNCIAS}

ABDELWAHAB, O. AMIN, N. K. Adsorption of phenol from aqueous solutions by Luffa cylindrica fibers: Kinetics, isotherm and thermodynamic studies. Egyptian Journal of Aquatic Research. 2014. 


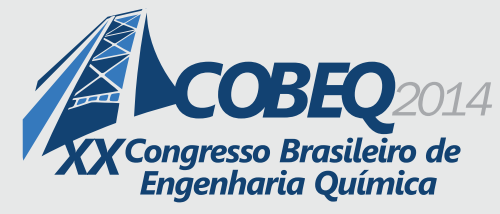

ARTHY M.; SARAVANAKUMAR M. P. Isotherm modeling, kinetic study and optimization of batch parameters for effective removal of Acid Blue 45 using tannery waste. Journal of Molecular Liquids, v. 187, p. 189-200, 2013.

BHATNAGAR, A.; SILLANPÄÄ, M.; Utilization of agro-industrial and municipal waste materials as potential adsorbents for water treatment - A review. Chemical Engineering Journal, v. 157 , p. $277-296,2010$.

BUSCA, G.; BERARDINELLI, S.; RESINI, C.; ARRIGHI, L. Technologies for the removal of phenol from fluid streams: a short review of recent developments, J. Hazard. Mater. 160 (2008) 265-288.

FERNANDES, R. Adsorventes alternativos para remoção de fenol em solução aquosa. Dissertação de Mestrado. Universidade Federal de Santa Catarina, Florianópolis. 2005.

FIERRO, V. TORNE'-FERNÁNDEZ, V. MONTANÉ, D. CELZARD, A. Adsorption of phenol onto activated carbons having different textural and surface properties. Science Direct. Microporous and Mesoporous Materials 111 (2008) 276-284.

KLEINÜBING, S. J. Remoção de cádmio, chumbo e cobre utilizando zeólita natural clinoptilolita em colunas de leito fixo. Dissertação de Mestrado. Universidade Estadual de Campinas, 2006.

MITTAL, A.; MITTAL, J.; MALVIYA, A.; KAUR,D.; GUPTA, V. K. Adsorption of hazardous dye crystal violet from wastewater by waste materials, J. Colloid Interface Sci., v. 343, p. 463473, 2010.

PALA, A.; TOKAT, E. Color removal from cotton textile industry wastewater in an activated sludge system with various additives. Water Research, v. 36, p. 2920-2925, 2002.

PARSONS, S. A.; JEFFERSONS, B. Introduction to Potable Water Treatment Process, $1^{\text {th }}$ ed., Blackwell Publishing, 2006.

RODRIGUES, L. A.; SILVA, M. L. C. P.; ALVARES-MENDES, M. O.; COUTINHO, A. R.; THIM, G. P. Phenol removal from aqueous solution by activated carbon produced from avocado kernel seeds. Chemical Engineering Journal, v. 174, p.49-57, 2011.

SANTO, C. M. E. A indústria de refinação de petróleo: Características e Tratamento das Águas Residuais. Laboratory of Separation and Reaction Engineering - LSRE. Departamento de Engenharia Química da Faculdade de Engenharia da Universidade do Porto, Portugal. 2010.

SINGH K. P.; MALIK A.; SINHA S.; OJHA P. Liquid-phase adsorption of phenols using activated carbons derived from agricultural waste material. Journal of Hazardous Materials, $v$. 150 , p. 626-641, 2008.

SOTO M. L.; MOURE A.; DOMINGUEZ H.; PARAJÓ J. C. Recovery, concentration and purification of phenolic compounds by adsorption- A review. Journal of Food Engineering, v.105, p.1-27, 2011.

ZOUBOULIS, A. I.; LAZARIDIS, N. K.; GROHMANN, A. Toxic metals removal from waste waters by upflow filtration with floating filter medium. I. The case of zinc. Separation Science and Technology, v. 37, p. 403-416, 2002. 